CPHT S 001.01.02

hep-ph/0202231

\title{
Hunting the QCD-Odderon in hard diffractive electroproduction of two pions
}

\author{
Ph. Hägler ${ }^{1}$, B. Pire ${ }^{2}$, L. Szymanowski ${ }^{2,3}$ and O.V. Teryaev ${ }^{4}$ \\ ${ }^{1}$ Institut für Theoretische Physik, Universität Regensburg, \\ D-93040 Regensburg, Germany \\ ${ }^{2}$ CPhT, École Polytechnique, F-91128 Palaiseau, France円 \\ ${ }^{3}$ Soltan Institute for Nuclear Studies, Hoża 69, \\ 00-681 Warsaw, Poland \\ ${ }^{4}$ Bogoliubov Lab. of Theoretical Physics, JINR, 141980 Dubna, Russia
}

October 23, 2018

\begin{abstract}
:
Charge asymmetries in diffractive electroproduction of two mesons are proportional to the interference of Pomeron and Odderon exchange amplitudes. We calculate in the framework of QCD and in the Born approximation a forward-backward charge asymmetry which turns out to be sizable in a kinematical domain accessible to HERA experiments. We predict a distinctive dependence of this asymmetry on the invariant mass of the two pions. Testing this prediction is a crucial step in the discovery of the QCD-Odderon.
\end{abstract}

\footnotetext{
${ }^{1}$ Unité mixte C7644 du CNRS.
} 
1. Pomeron and Odderon exchanges are the theoretically dominant contributions to hadronic cross sections at high energy. They appear on an equal footing in the QCD description of hadronic reactions, and in the lowest order approximation they correspond to colour singlet exchanges in the $t$-channel with two and three gluons, respectively.

The relevance of the Odderon exchange for hadronic reactions was emphasized long ago [1]. In perturbative QCD the Odderon is described by the Bartels-KwiecinskiPraszalowicz (BKP) equation [2]. In spite of many attempts to solve the BKP equation, its solutions are still known only partially although much progress has been recently achieved [3, 田, 5, 6]. Still this appears to be in a strong contrast with the QCD description of the Pomeron, where solutions of the leading order Balitsky-Fadin-Kuraev-Lipatov (BFKL) equation are well known [7].

The Odderon remains a mistery also from an experimental point of view. On the one hand, recent studies of the elastic $p p$ scattering show that one needs the Odderon contribution to understand the data in the dip region [13]. On the other hand, the studies of reactions which should select only the odderon exchange didn't show any clear signal of its importance. In the case of diffractive $\eta_{c}$-meson photoproduction, the QCD prediction in the Born approximation for the cross section is rather small [8, 9]. The inclusion of evolution following from the BKP equation [10] leads to an increase of the predicted cross section for this process by one order of magnitude. Recent experimental studies at HERA of exclusive $\pi^{0}$ photoproduction [1] also indicate a very small cross section for this process, in disagreement with theoretical predictions based on the stochastic vacuum model [12]. In all these meson production processes the scattering amplitude describing Odderon exchange enters quadratically in the cross section.

In 14] it was suggested to study Odderon effects at the amplitude level by means of the asymmetries in open charm production. Since the final state quark-antiquark pair has no definite charge parity both Pomeron and Odderon exchanges contribute to this process. The Odderon amplitude enters linearly in the asymmetries and therefore one can hope that Odderon effects can show up more easily. Moreover, the difficulty with the understanding of soft processes in QCD calls for studies of Odderon contributions in hard processes, such as electroproduction, where factorization properties allow to perturbatively calculate a short-distance part of the scattering amplitude.

In this paper, we propose to take advantage of a number of interesting features of the two pion diffractive electroproduction process to search for the QCD-Odderon at the amplitude level. Here again the two pion state doesn't have any definite charge parity and both Pomeron and Odderon exchanges contribute. The authors of Ref. [15] suggest to study the charge asymmetry in soft photoproduction of two pions to select the interference of the two amplitudes. Our work shares a number of features with this work. The originality of our study of the electroproduction process is to work in a perturbative QCD framework which enables us to derive well founded predictions in an accessible kinematical domain. 


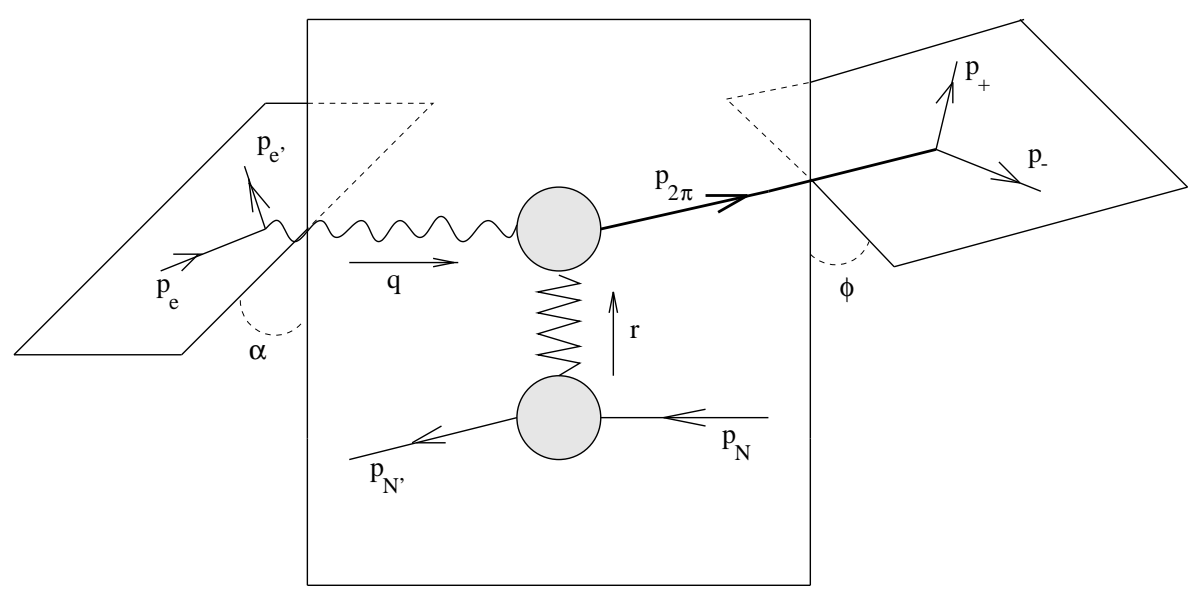

Figure 1: Kinematics of the electroproduction of two pions

The aim of the present paper is to study the charge asymmetries in the reaction

$$
e^{-}\left(p_{e}\right) p\left(p_{N}\right) \rightarrow e^{-}\left(p_{e}^{\prime}\right) \pi^{+}\left(p_{+}\right) \pi^{-}\left(p_{-}\right) p^{\prime}\left(p_{N}^{\prime}\right)
$$

within perturbative QCD, see Fig. 1. This includes the convolution of perturbatively calculable hard subprocess with the two non-perturbative inputs : 2-pion generalized distribution amplitude (GDA) and Pomeron-Odderon (P/O) proton impact factors. GDA [17] are just the light-cone wave functions of the two pion system. Contrary to, say, $\rho$ meson wave functions, they do not require the selection of a particular charge parity and so are ideally suited for studies of the $\mathrm{P} / \mathrm{O}$ interference. In order to justify the use of perturbation theory for this process we consider the electroproduction of this system in which the hard scale is supplied by the squared mass $-Q^{2}$ of the virtual photon, $Q^{2}$ being of the order of a few $\mathrm{GeV}^{2}$.

We intend to study the dominant (for asymptotically large $Q^{2}$ ) contribution to the asymmetries. This corresponds to the process with longitudinally polarized photon. In this case the "longitudinal part" of the two pion wave function is selected, which is a straightforward generalization of the longitudinal polarization of vector meson. The longitudinal polarization vector is enhanced by a Lorentz boost, together with the crosssection of longitudinally polarized pion pair production. This is the twist-2 contribution of the subprocess $\gamma^{*} \mathrm{P} / \mathrm{O} \rightarrow q \bar{q} \rightarrow \pi^{+} \pi^{-}$, i.e. in the case of the collinear factorization for the $q \bar{q} \rightarrow \pi^{+} \pi^{-}$transition. At moderate $Q^{2}$ it is customary to take into account the transverse momentum $k_{\perp}$ dependence of the meson wave functions which is a model way of accounting for higher twist contributions, so that for growing $Q^{2}$ the result is undistinguishible from the one of standard collinear factorization ?. The relation between the initial and final polarization states is due to the fact that for high-energy diffractive processes s-channel helicity conservation is quite well satisfied. Since the cross sections $\sigma_{L}$ and $\sigma_{T}$ for the longitudinal and transverse photon polarization, respectively, are of the same order of magnitude at moderate $Q^{2}$ [16], an experimental separation of $\sigma_{L}$ is highly desirable before confronting our predictions with data.

\footnotetext{
${ }^{2}$ We postpone the consideration of $k_{\perp}$-dependence for future work.
} 

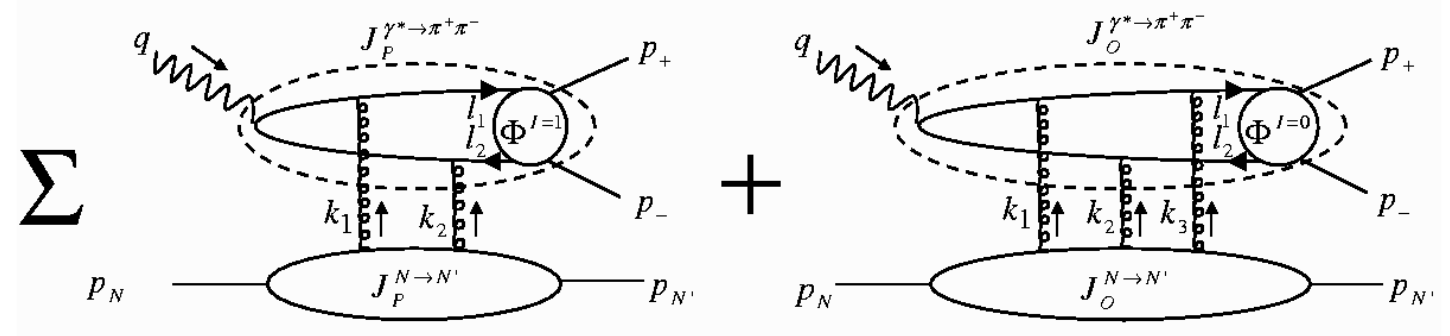

Figure 2: Feynman diagrams describing $\pi^{+} \pi^{-}$electroproduction in the Born approximation

Since the transverse polarization of the pion pair is the only source of the amplitude dependence on the azimuthal angle of the pions in their c.m. frame, the amplitudes and cross sections are independent of this angle in our approximation. As a result, the transverse charge asymmetry, resulting from the distribution in this angle and discussed in [15], is zero. Due to that restriction, we only study the forward-backward charge asymmetry.

In the present study we calculate the lowest perturbative order contribution to the charge asymmetry, i.e. without taking into account the evolution following from the BFKL or the BKP equation. Our results should be therefore treated as an estimate of the asymmetries. The above mentioned evolutions can be included into the scattering amplitudes in a similar way as in Ref. [10].

2. The basic object necessary to calculate the charge asymmetry is the scattering amplitude for the process with a longitudinal virtual photon (Fig. 2).

$$
\gamma_{L}^{*}(q) p\left(p_{N}\right) \rightarrow \pi^{+}\left(p_{+}\right) \pi^{-}\left(p_{-}\right) p^{\prime}\left(p_{N^{\prime}}\right) .
$$

We introduce a Sudakov representation with the Sudakov momenta $p_{1}, p_{2}$ obeying the equation $s=2 p_{1} \cdot p_{2}$, where $s$ is related to the total energy squared of the virtual photon - proton system, $Q^{2}$ and the proton target mass $M$ as

$$
\left(q+p_{N}\right)^{2}=s-Q^{2}+M^{2} \approx s
$$

we get for the virtual photon momentum :

$$
q^{\mu}=p_{1}^{\mu}-\frac{Q^{2}}{s} p_{2}^{\mu}
$$

and for the momentum of the two pion system :

$$
p_{2 \pi}^{\mu}=\left(1-\frac{\vec{p}_{2 \pi}^{2}}{s}\right) p_{1}^{\mu}+\frac{m_{2 \pi}^{2}+\vec{p}_{2 \pi}^{2}}{s} p_{2}^{\mu}+p_{2 \pi \perp}^{\mu}, \quad p_{2 \pi \perp}^{2}=-\vec{p}_{2 \pi}^{2}
$$

The quark $\left(l_{1}\right)$ and antiquark $\left(l_{2}\right)$ momenta inside the loop before forming two pion system are parametrized as :

$$
l_{1}^{\mu}=z p_{1}^{\mu}+\frac{m^{2}+\left(\vec{l}+z \vec{p}_{2 \pi}\right)^{2}}{z s} p_{2}^{\mu}+\left(l_{\perp}+z p_{2 \pi \perp}\right)^{\mu}
$$




$$
l_{2}^{\mu}=\bar{z} p_{1}^{\mu}+\frac{m^{2}+\left(-\vec{l}+\bar{z} \vec{p}_{2 \pi}\right)^{2}}{\bar{z} s} p_{2}^{\mu}+\left(-l_{\perp}+\bar{z} p_{2 \pi \perp}\right)^{\mu}
$$

where $2 \vec{l}$ is the relative transverse momentum of the quarks forming the two pion system and $\bar{z}=1-z$, up to small corrections of the order $\vec{p}_{2 \pi}^{2} / s$. The collinear approximation of the factorization procedure of the description of the two pion formation through the generalized distribution amplitude leads to the vector $\vec{l}=\overrightarrow{0}$ in the hard amplitude.

In a similar way as in (5), (6) we parametrize the momenta of produced pions as

$$
\begin{gathered}
p_{+}^{\mu}=\zeta p_{1}^{\mu}+\frac{m_{\pi}^{2}+\left(\vec{p}+\zeta \vec{p}_{2 \pi}\right)^{2}}{\zeta s} p_{2}^{\mu}+\left(p_{\perp}+\zeta p_{2 \pi \perp}\right)^{\mu} \\
p_{-}^{\mu}=\bar{\zeta} p_{1}^{\mu}+\frac{m_{\pi}^{2}+\left(-\vec{p}+\bar{\zeta} \vec{p}_{2 \pi}\right)^{2}}{\bar{\zeta} s} p_{2}^{\mu}+\left(-p_{\perp}+\bar{\zeta} p_{2 \pi \perp}\right)^{\mu}
\end{gathered}
$$

where $2 \vec{p}$ is now the relative transverse momentum of the produced pions, $\zeta=\frac{p_{2} \cdot p_{+}}{p_{2} \cdot p_{2 \pi}}$ is the fraction of the longitudinal momentum $p_{2 \pi}$ carried by the produced $\pi^{+}$, and $\bar{\zeta}=1-\zeta$. The variable $\zeta$ is related to the polar decay angle $\theta$ defined in the rest frame of the pion pair by

$$
\beta \cos \theta=2 \zeta-1, \quad \beta \equiv \sqrt{1-\frac{4 m_{\pi}^{2}}{m_{2 \pi}^{2}}}
$$

Since the "longitudinal part" of the two pion wave function depends only on the angle $\theta$ and doesn't depend on the azimuthal decay angle $\phi$ (in the same rest frame of the pair) we focus on the calculation of forward-backward asymmetries expressed in terms of $\theta$ (see below).

The nucleon momentum in the initial state is given by

$$
p_{N}^{\mu}=p_{2}^{\mu}+\frac{M^{2}}{s} p_{1}^{\mu} .
$$

The squared momentum transfer $t=r^{2}\left(r^{\mu}=p_{2 \pi}^{\mu}-q^{\mu}\right)$ can be written as

$$
t=r^{2}=-\vec{p}_{2 \pi}^{2}+t_{m i n}, \quad t_{m i n}=-\frac{M^{2}\left(Q^{2}+m_{2 \pi}^{2}\right)^{2}}{s^{2}}
$$

3. It is well known (see e.g. [9] and references therein) that for large values of $s$ and small momentum transfer $t$ the scattering amplitudes can be represented as convolutions over the two-dimensional transverse momenta of the $t$-channel gluons.

For the Pomeron exchange, which corresponds in the Born approximation to the exchange of two gluons in a colour singlet state, the impact representation has the form:

$$
\mathcal{M}_{P}=-i s \int \frac{d^{2} \vec{k}_{1} d^{2} \vec{k}_{2} \delta^{(2)}\left(\vec{k}_{1}+\vec{k}_{2}-\vec{p}_{2 \pi}\right)}{(2 \pi)^{2} \vec{k}_{1}^{2} \vec{k}_{2}^{2}} J_{P}^{\gamma^{*} \rightarrow \pi^{+} \pi^{-}}\left(\vec{k}_{1}, \vec{k}_{2}\right) \cdot J_{P}^{N \rightarrow N^{\prime}}\left(\vec{k}_{1}, \vec{k}_{2}\right)
$$

where $J_{P}^{\gamma^{*} \rightarrow \pi^{+} \pi^{-}}\left(\vec{k}_{1}, \vec{k}_{2}\right)$ and $J_{P}^{N \rightarrow N^{\prime}}\left(\vec{k}_{1}, \vec{k}_{2}\right)$ are the impact factors for transition $\gamma^{*} \rightarrow \pi^{+} \pi^{-}$via Pomeron exchange and of the nucleon in initial state $N$ into the nucleon in the final state $N^{\prime}$. 
The corresponding representation for the Odderon exchange, i.e. the exchange of three gluons in a colour singlet state, is given by the formula

$$
\mathcal{M}_{O}=-\frac{8 \pi^{2} s}{3 !} \int \frac{d^{2} \vec{k}_{1} d^{2} \vec{k}_{2} d^{2} \vec{k}_{3} \delta^{(2)}\left(\vec{k}_{1}+\vec{k}_{2}+\vec{k}_{3}-\vec{p}_{2 \pi}\right)}{(2 \pi)^{6} \vec{k}_{1}^{2} \vec{k}_{2}^{2} \vec{k}_{3}^{2}} J_{O}^{\gamma^{*} \rightarrow \pi^{+} \pi^{-}} \cdot J_{O}^{N \rightarrow N^{\prime}}
$$

where $J_{O}^{\gamma^{*} \rightarrow \pi^{+} \pi^{-}}\left(\vec{k}_{1}, \vec{k}_{2}, \vec{k}_{3}\right)$ and $J_{O}^{N \rightarrow N^{\prime}}\left(\vec{k}_{1}, \vec{k}_{2}, \vec{k}_{2}\right)$ are the impact factors for the transition $\gamma^{*} \rightarrow \pi^{+} \pi^{-}$via Odderon exchange and of the nucleon in initial state $N$ into the nucleon in the final state $N^{\prime}$.

The impact factors are calculated by the standard methods, see e.g. Ref. 18 and references therein. An important aspect of the present study is the inclusion of an appropriate two pion distribution amplitude which we now discuss.

4. The two-pion generalized distribution amplitude (GDA) [17, 19] contains the full strong interactions between the two pions. So far no experimental information exists on the two-pion GDA. Watson's theorem imposes that the dynamical phases of the twopion GDA are identical to the phase shifts in elastic $\pi \pi$ scattering as long as $m_{2 \pi}$ is below the inelastic threshold. This relation may be used as an input for a model GDA in the $m_{2 \pi}$-region up to $1 \mathrm{GeV}$. For higher values of $m_{2 \pi}$ we assume that the phase is still approximately equal to the $\pi \pi$ phase shift. The Odderon exchange process involves the production of a pion pair in the $C$-even channel which corresponds to even isospin. In the numerical studies we will use a simple ansatz [20] for the isosinglet distribution amplitude $\Phi^{I=0}\left(z, \zeta, m_{2 \pi}^{2}\right)$, in a slightly enlarged $m_{2 \pi}$ range. We only consider the contributions from $u$ - and $d$-quarks, i.e. we take $n_{f}=2$.

A crucial point is the choice of the parametrization of the phases in the GDA's. Let us discuss first the isosinglet $s-$ and $d$-wave phase shifts, $\delta_{0}$ and $\delta_{2}$. Through interference effects, the rapid variation of a phase shift leads to a characteristic $m_{2 \pi}$-dependence of the asymmetry. We use

$$
\begin{aligned}
& \Phi^{I=0}\left(z, \zeta, m_{2 \pi}\right)=10 z(1-z)(2 z-1) R_{\pi} \\
& {\left[-\frac{3-\beta^{2}}{2} e^{i \delta_{0}\left(m_{2 \pi}\right)}\left|B W_{f_{0}}\left(m_{2 \pi}^{2}\right)\right|+\beta^{2} e^{i \delta_{2}\left(m_{2 \pi}\right)}\left|B W_{f_{2}}\left(m_{2 \pi}^{2}\right)\right| P_{2}(\cos \theta)\right],}
\end{aligned}
$$

with $R_{\pi}=0.5$ and $\beta$ given by Eq. (9). In our studies we fix the shapes of the phase shifts $\delta_{0}$ and $\delta_{2}$ from a fit to data presented in [21]. Let us stress that the exact description of the phase shifts is crucial for our analysis. It is well known that the Breit-Wigner parametrization of the amplitude is only a reasonable approximation in the vicinity of the resonance peaks. In particular the vicinity of the $K \bar{K}$-threshold leads to some theoretical uncertainty of our results, although we do not expect a dramatic effect.

$\left|B W_{f}\left(m_{2 \pi}^{2}\right)\right|$ is the modulus of the Breit-Wigner amplitudes

$$
\begin{gathered}
B W_{f_{0}}\left(m_{2 \pi}^{2}\right)=\frac{m_{f_{0}}^{2}}{m_{f_{0}}^{2}-m_{2 \pi}^{2}-i m_{f_{0}} \Gamma_{f_{0}}}, \quad m_{f_{0}}=980 \mathrm{MeV}, \quad \Gamma_{f_{0}}=50-100 \mathrm{MeV} \\
B W_{f_{2}}\left(m_{2 \pi}^{2}\right)=\frac{m_{f_{2}}^{2}}{m_{f_{2}}^{2}-m_{2 \pi}^{2}-i m_{f_{2}} \Gamma_{f_{2}}}, \quad m_{f_{2}}=1275 \mathrm{MeV}, \quad \Gamma_{f_{2}}=186 \mathrm{MeV} .
\end{gathered}
$$


To take into account of the uncertainty in the $f_{0}$ width, we will present results for the two extreme allowed values.

The Pomeron exchange process involves the production of a pion pair in the $C$-odd state. Its amplitude can be fully computed for values of $m_{2 \pi}$ where the timelike electromagnetic pion form factor $F_{\pi}\left(m_{2 \pi}^{2}\right)$ is known provided the lowest Gegenbauer polynomial component is dominant. The modulus of $F_{\pi}$ has been well measured in the process $e^{+} e^{-} \rightarrow \pi^{+} \pi^{-}$. By Watson's theorem its phase is equal to the $p$-wave phase shift $\delta_{1}$, provided that $m_{2 \pi}$ is in the range where $\pi \pi$ scattering is elastic. This is rather well satisfied for values of $m_{2 \pi}$ up to $1 \mathrm{GeV}$. We assume that for $m_{2 \pi}$ up to $1.5 \mathrm{GeV}$ we can still use the phase shifts from $\pi \pi$ elastic scattering as the phase of the distribution amplitude. In our numerical studies we take a $F_{\pi}$-parameterization inspired by the $N=1$ model of Ref. 22

$$
\Phi^{I=1}\left(z, \zeta, m_{2 \pi}\right)=6 z(1-z) \beta \cos \theta F_{\pi}\left(m_{2 \pi}^{2}\right)
$$

where

$$
F_{\pi}\left(m_{2 \pi}^{2}\right)=\frac{1}{(1-0.145)} B W_{\rho} \frac{1+1.85 \cdot 10^{-3} \cdot B W_{\omega}}{1+1.85 \cdot 10^{-3}}
$$

with

$$
\begin{aligned}
& B W_{\rho}\left(m_{2 \pi}^{2}\right)=\frac{m_{\rho}^{2}}{m_{\rho}^{2}-m_{2 \pi}^{2}-i \sqrt{m_{2 \pi}^{2}} \Gamma_{\rho}\left(m_{2 \pi}^{2}\right)}, \\
& \Gamma_{\rho}\left(m_{2 \pi}^{2}\right)=\Gamma_{\rho} \frac{m_{\rho}^{2}}{m_{2 \pi}^{2}} \frac{\left(m_{2 \pi}^{2}-4 m_{\pi}^{2}\right)^{3 / 2}}{\left(m_{\rho}^{2}-4 m_{\pi}^{2}\right)^{3 / 2}}, \quad m_{\rho}=773 \mathrm{MeV}, \quad \Gamma_{\rho}=145 \mathrm{MeV},
\end{aligned}
$$

and

$$
B W_{\omega}\left(m_{2 \pi}^{2}\right)=\frac{m_{\omega}^{2}}{m_{\omega}^{2}-m_{2 \pi}^{2}-i m_{\omega} \Gamma_{\omega}}, \quad m_{\omega}=782 \mathrm{MeV}, \quad \Gamma_{\omega}=8.5 \mathrm{MeV} .
$$

The parametrization of the pion form-factor given by Eq. (18) leads to a reasonable description of the data on the square of the pion form-factor, see [22]. It describes also satisfactorily the $p$-wave phase shift in the region of $2 \pi$ invariant mass smaller than 1.5 $\mathrm{GeV}$. Above $1 \mathrm{GeV}$ the phase shift given by the original parametrization of Ref. [22] strongly overestimates the data points presented in [21], due to a problematic $\rho^{\prime}(1370)-$ contribution. Since a correct treatment of phases is crucial for our predictions, we do not include any $\rho^{\prime}-$ contribution.

5. After choosing the two pion distribution amplitude, the calculation of the necessary impact factors is straightforward. Skipping unessential details, let us now present the final results. For the longitudinal polarization of virtual photon $\gamma_{L}^{*}$ we obtain for the impact-factor for $\gamma_{L}^{*} \rightarrow \pi^{+} \pi^{-}$the following formulas:

- Pomeron exchange:

$$
J_{P}^{\gamma_{L}^{*} \rightarrow \pi^{+} \pi^{-}}\left(\vec{k}_{1}, \vec{k}_{2}\right)=-\frac{i e g^{2} \delta^{a b} Q}{2 N_{C}} \int_{0}^{1} d z z \bar{z} P_{P}\left(\vec{k}_{1}, \vec{k}_{2}\right) \Phi^{I=1}\left(z, \zeta, m_{2 \pi}^{2}\right)
$$




$$
P_{P}\left(\vec{k}_{1}, \vec{k}_{2}\right)=\frac{1}{z^{2} \vec{p}_{2 \pi}^{2}+\mu^{2}}+\frac{1}{\bar{z}^{2} \vec{p}_{2 \pi}^{2}+\mu^{2}}-\frac{1}{\left(\vec{k}_{1}-z \vec{p}_{2 \pi}\right)^{2}+\mu^{2}}-\frac{1}{\left(\vec{k}_{1}-\bar{z} \vec{p}_{2 \pi}\right)^{2}+\mu^{2}}
$$

where $\vec{k}_{1}+\vec{k}_{2}=\vec{p}_{2 \pi}$ and $\mu^{2}=m_{q}^{2}+z \bar{z} Q^{2}$.

- Odderon exchange (where $\vec{k}_{1}+\vec{k}_{2}+\vec{k}_{3}=\vec{p}_{2 \pi}$ ) :

$$
\begin{gathered}
J_{O}^{\gamma_{L}^{*} \rightarrow \pi^{+} \pi^{-}}\left(\vec{k}_{1}, \vec{k}_{2}, \vec{k}_{3}\right)=-\frac{i e g^{3} d^{a b c} Q}{4 N_{C}} \int_{0}^{1} d z z \bar{z} P_{O}\left(\vec{k}_{1}, \vec{k}_{2}, \vec{k}_{3}\right) \frac{1}{3} \Phi^{I=0}\left(z, \zeta, m_{2 \pi}^{2}\right) \\
P_{O}\left(\vec{k}_{1}, \vec{k}_{2}, \vec{k}_{3}\right)=\frac{1}{z^{2} \vec{p}_{2 \pi}^{2}+\mu^{2}}-\frac{1}{\bar{z}^{2} \vec{p}_{2 \pi}^{2}+\mu^{2}} \\
-\sum_{i=1}^{3}\left(\frac{1}{\left(\vec{k}_{i}-z \vec{p}_{2 \pi}\right)^{2}+\mu^{2}}-\frac{1}{\left(\vec{k}_{i}-\bar{z}_{2 \pi}\right)^{2}+\mu^{2}}\right)
\end{gathered}
$$

The value of the strong coupling constant $g$ in the hard block is assumed to correspond to the 1-loop running coupling constant with $n_{f}=2, \alpha_{s}\left(Q^{2}\right)=\frac{g^{2}}{4 \pi}=12 \pi /\left[29 \ln \left(\frac{Q^{2}}{\Lambda_{Q C D}^{2}}\right)\right]$. In order to estimate the theoretical error we vary the value of $\Lambda_{Q C D}=0.2-0.35 \mathrm{GeV}$. This does not lead to any dramatic change in the magnitude of the asymmetry.

Finally we have to fix the soft parts of our amplitudes, i.e. the proton impact factors. They cannot be calculated within perturbation theory. In our estimates we will use phenomenological, eikonal models of these impact factors proposed in Refs. [23] and [24]. We take

- for the Pomeron exchange :

$$
J_{P}^{N \rightarrow N^{\prime}}=i \frac{\bar{g}^{2} \delta^{a b}}{2 N_{C}} 3\left[\frac{A^{2}}{A^{2}+1 / 2 \vec{p}_{2 \pi}^{2}}-\frac{A^{2}}{A^{2}+1 / 2\left(\vec{k}_{1}^{2}+\vec{k}_{2}^{2}\right)}\right],
$$

- for the Odderon exchange :

$$
J_{O}^{N \rightarrow N^{\prime}}=-i \frac{\bar{g}^{3} d^{a b c}}{4 N_{C}} 3\left[F\left(\vec{p}_{2 \pi}, 0,0\right)-\sum_{i=1}^{3} F\left(\vec{k}_{i}, \vec{p}_{2 \pi}-\vec{k}_{i}, 0\right)+2 F\left(\vec{k}_{1}, \vec{k}_{2}, \vec{k}_{3}\right)\right]
$$

where

$$
F\left(\vec{k}_{1}, \vec{k}_{2}, \vec{k}_{3}\right)=\frac{A^{2}}{A^{2}+\frac{1}{2}\left[\left(\vec{k}_{1}-\vec{k}_{2}\right)^{2}+\left(\vec{k}_{2}-\vec{k}_{3}\right)^{2}+\left(\vec{k}_{3}-\vec{k}_{1}\right)^{2}\right]}
$$

and $A=\frac{m_{\rho}}{2}$. 
In these formula, we have denoted the QCD-coupling constant as $\bar{g}$. While it is natural to take $Q^{2}$ as the scale of the strong coupling constant in the upper impact factor, a typical hadronic scale $M^{2}$ is better suited for the lower one. The value of the coupling constant $\bar{g}=g\left(M^{2}\right)$ is one of the main sources of theoretical uncertainties of our numerical results.

In the original Refs. [23, 24] it was assumed that $\alpha_{\text {soft }}=\frac{\bar{g}^{2}}{4 \pi} \approx 1$. In view of the results of the recent studies [12], it seems that this value is too large for a correct description of the $p p$ differential cross-section in the region of the dip, and one should rather take $\alpha_{\text {soft }}=0.3-0.7$. In order to visualize this rather large uncertainty we present our results with an error band corresponding to this interval of $\alpha_{\text {soft }}$. We also want to emphasize that an increase of the value of $\alpha_{\text {soft }}$ to $\alpha_{\text {soft }} \approx 1$ raises our predictions for the asymmetry by a factor $\approx 1.3$.

6. Let us now present our estimates of the charge asymmetry. We define the forward - backward asymmetry as

$$
\begin{array}{r}
A\left(Q^{2}, t, m_{2 \pi}^{2}\right)=\frac{\int \cos \theta d \sigma\left(s, Q^{2}, t, m_{2 \pi}^{2}, \theta\right)}{\int d \sigma\left(s, Q^{2}, t, m_{2 \pi}^{2}, \theta\right)} \\
=\frac{\int_{-1}^{1} \cos \theta d \cos \theta 2 \operatorname{Re}\left[\mathcal{M}_{P}^{\gamma_{L}^{*}}\left(\mathcal{M}_{O}^{\gamma_{L}^{*}}\right)^{*}\right]}{\int_{-1}^{1} d \cos \theta\left[\left|\mathcal{M}_{P}^{\gamma_{L}^{*}}\right|^{2}+\left|\mathcal{M}_{O}^{\gamma_{L}^{*}}\right|^{2}\right]}
\end{array}
$$

which may be rewritten as

$$
A\left(Q^{2}, t, m_{2 \pi}^{2}\right)=\frac{\int_{(1-\beta) / 2}^{(1+\beta) / 2} d \zeta \frac{1}{\beta}(2 \zeta-1) 2 \operatorname{Re}\left[\mathcal{M}_{P}^{\gamma_{L}^{*}}\left(\mathcal{M}_{O}^{\gamma_{L}^{*}}\right)^{*}\right]}{\int_{(1-\beta) / 2}^{(1+\beta) / 2} d \zeta\left[\left|\mathcal{M}_{P}^{\gamma_{L}^{*}}\right|^{2}+\left|\mathcal{M}_{O}^{\gamma_{L}^{*}}\right|^{2}\right]}
$$

Instead of weighted integration of the cross-section it is possible to perform a full angular analysis. The numerator of the asymmetry would then be provided by the $\cos \theta-$ term which is characteristic of the longitudinal polarization of the pion pair, while in the denominator one may extract experimentally the contribution of the longitudinally polarized pion pair in complete analogy to the case of longitudinally polarized vector mesons.

We checked that the squared Odderon contribution in the denominator can be neglected, so that the asymmetry is practically a measure of the ratio of the Odderon and the Pomeron amplitudes.

There is no $s$-dependence in our framework, within the approximation which we make, provided $s$ is large enough for the usual high energy approximation to hold. The charge asymmetry is plotted in Figs. 3 and 4 as a function of the two pion invariant mass $m_{2 \pi}$. The main characteristic is the high numerical value that we get for values of $m_{2 \pi}$ around the $f_{0}$ mass. This is in strong contrast to the result obtained in a completely 


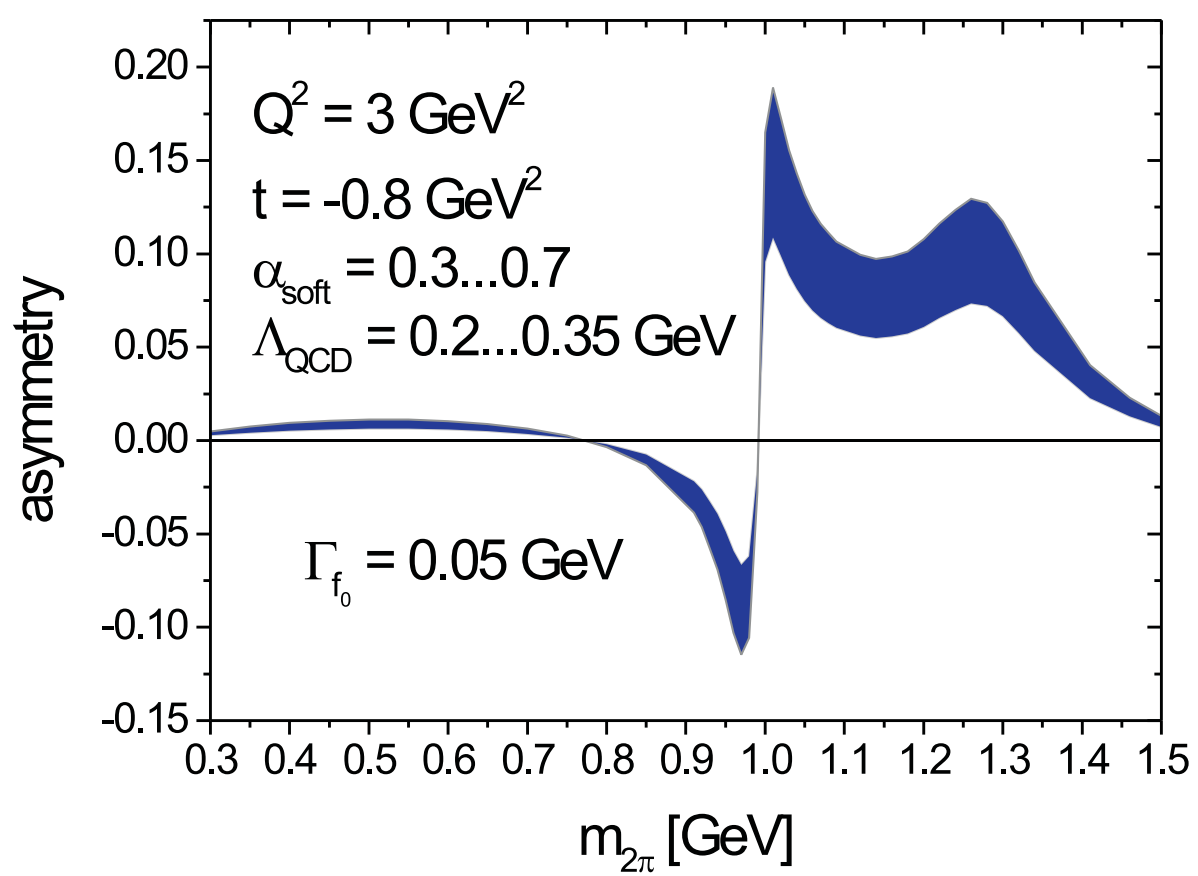

Figure 3: Charge asymmetry given by Eq. (28) for a minimal $f_{0}$ width, with an error band showing the uncertainty comming from different values of $\alpha_{\text {soft }}$ and $\Lambda_{Q C D}$.

different framework by the authors of Ref. [15]. The characterisic $m_{2 \pi}$ dependence in Fig. 3 and Fig. 4 is completely understood in terms of the $\pi \pi$ phase shifts and the factor $\sin \left(\delta^{0}-\delta^{1}\right)$. The phase difference vanishes for $m_{2 \pi} \approx 0.75 \mathrm{GeV}$ and $m_{2 \pi} \approx 1 \mathrm{GeV}$ resulting in two zeros of the charge asymmetry. The magnitude of the asymmetry depends much on the width of the $f_{0}$ meson which is estimated to be $50-100 \mathrm{MeV}$. The curve shown in the Fig. 3 and in the Fig. 4 are obtained with the extreme values of this width. The $d$-wave contribution shows up around $1.3 \mathrm{GeV}$ on Figs. 3, 4 and 5. In Fig. 5 we show also the $Q^{2}$ dependence of the asymmetry which turns out to be rather moderate.

The $t$ dependence of the asymmetry, which is plotted in Fig. 6, is quite interesting since it has a characteristic zero around $t=-0.1 \mathrm{GeV}^{2}$. This zero in the odderon amplitude has already been discussed in Ref. [10]. The practical outcome of this $t$ dependence is that it is better to focus on moderate values of $t$ and, if an integration over $t$ is needed in order to improve the statistics, to choose at least $-t \approx 0.1 \mathrm{GeV}^{2}$ as a lower integration limit.

7. Let us now comment on the possible theoretical uncertainties of our calculations.

- Higher twist contributions and corrections to the calculated expressions of order $m_{2 \pi}^{2} / Q^{2}$ or $t / Q^{2}$ may well be non negligible. For instance, $k_{\perp}$ effects in the $\pi^{+} \pi^{-}$ wave function mentioned above may be important at relatively low $Q^{2}$ [25] as well as the effects of transversely polarized photon. An estimate of these corrections is certainly desirable but clearly out of the scope of the present paper.

- $\mathrm{QCD}$ evolution à la BFKL and $\ln \left(s / \Lambda_{Q C D}^{2}\right)$ corrections may be calculated for the Pomeron and Odderon exchanges as well as the $\ln \left(Q^{2} / \Lambda_{Q C D}^{2}\right)$ corrections for the 


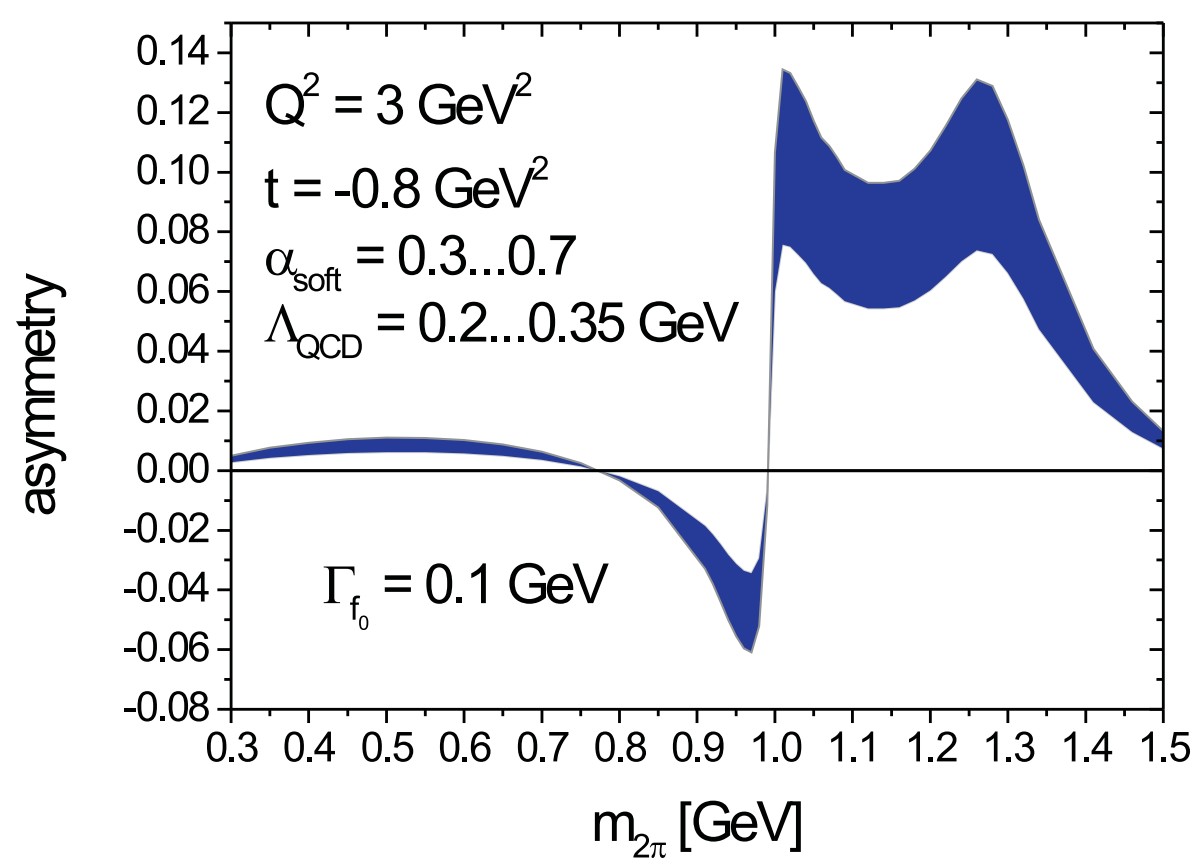

Figure 4: Same as Fig. 3 but for maximal $f_{0}$ width.

generalized distribution amplitudes. We expect that they do not have drastic effects on ratios such as the charge asymmetry which we have calculated. This may be controlled in the future.

- The exact values of the scales of the coupling constants should be determined by the presently unknown higher order corrections in the hard and soft parts, respectively. It is interesting that in the case of the dominance of Abelian contributions to radiative corrections, the coupling renormalization would be reduced, by use of the Ward identities, to bubble insertions into the gluon line, so that the scales would be common for the hard and soft parts and determined by the gluon off-shellness. Due to the integration over the gluon momenta the latter should be averaged to some intermediate value between $Q^{2}$ and $M^{2}$, so that the above naive estimate may still be valid.

- The $\pi \pi$ distribution amplitude is a non perturbative object which we certainly do not exactly know. Although its phase is theoretically under control in the lower mass range, its magnitude and its $z$ - and $\zeta$ - dependence may be quite different from the simple ansatz that we have adopted. Let us stress however that parts of this uncertainty can be resolved by other experiments, namely two pion production in $e \gamma$ collisions [20] and in ep collisions at medium energies [26] sensitive to the C-even and C-odd components of GDA.

Let us now briefly indicate possible future studies closely related to the approach that we have developed.

- Single spin asymmetries should show up in the same reaction [27. Longitudinally polarized electron beams are providing circularly polarized virtual photons. In turn, 


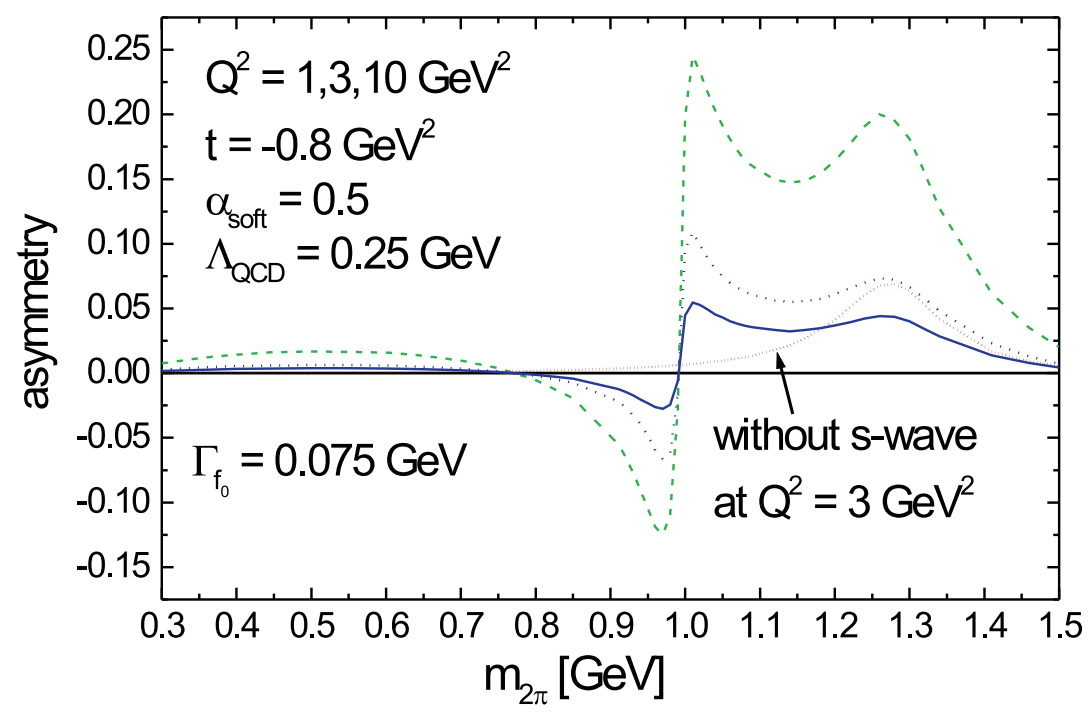

Figure 5: $m_{2 \pi}$-dependence of the asymmetry for $t=-.8 \mathrm{GeV}^{2}$ and for different values of $Q^{2}$ : $1 \mathrm{GeV}^{2}$ (dashed line), $3 \mathrm{GeV}^{2}$ (dotted line), $10 \mathrm{GeV}^{2}$ (solid line); the $d$-wave contribution at $Q^{2}=3 \mathrm{GeV}^{2}$ is shown with a dense dotted line; the $f_{0}$ width has been taken to be $75 \mathrm{MeV}$.

they give rise to single spin azimuthal asymmetries, similar to those appearing in the $\gamma^{*} \gamma$ production of pion pairs [20] and triplets [28]. The expression for the asymmetry is similar to the one for the charge asymmetry with the notable difference that the imaginary, instead of real part of the relevant product of amplitudes appears in the numerator of the analog of Eq.28. The consequence is that, in the case of the Pomeron-Odderon interference, whose amplitudes are mostly imaginary and real, respectively, there appears a factor $\cos \left(\delta_{0}-\delta_{1}\right)$ instead of $\sin \left(\delta_{0}-\delta_{1}\right)$, which leads to a completely different $m_{2 \pi}$ dependence. Note that in the case of the calculations of the single spin asymmetry one has to take into account transverse polarizations of a photon.

- Charge asymmetries can also be studied in the interesting case of $\gamma^{*} \gamma^{*}$ scattering which may be measured at $e^{+} e^{-}$colliders. A specific feature of this case is that both impact factors are calculable within perturbative QCD.

8. In conclusion, let us stress that we have demonstrated that the understanding of diffractive processes within perturbative QCD is bound to the discovery of sizable charge asymmetries in electroproduction of two charged mesons.

We applied the powerful tool of QCD factorization when the hard part (subprocess) is calculated perturbatively, while the soft ingredients (GDA and proton impact factor) should be modeled or, better, measured, which poses a new challenging problem for experimentalists,

Data on this reaction in the kinematical domain suitable for our calculation (i.e. large $s$, small $t, Q^{2}$ above $1 \mathrm{GeV}^{2}$ and $m_{2 \pi}$ below $1.5 \mathrm{GeV}$ ) should be easy to get and analyze 


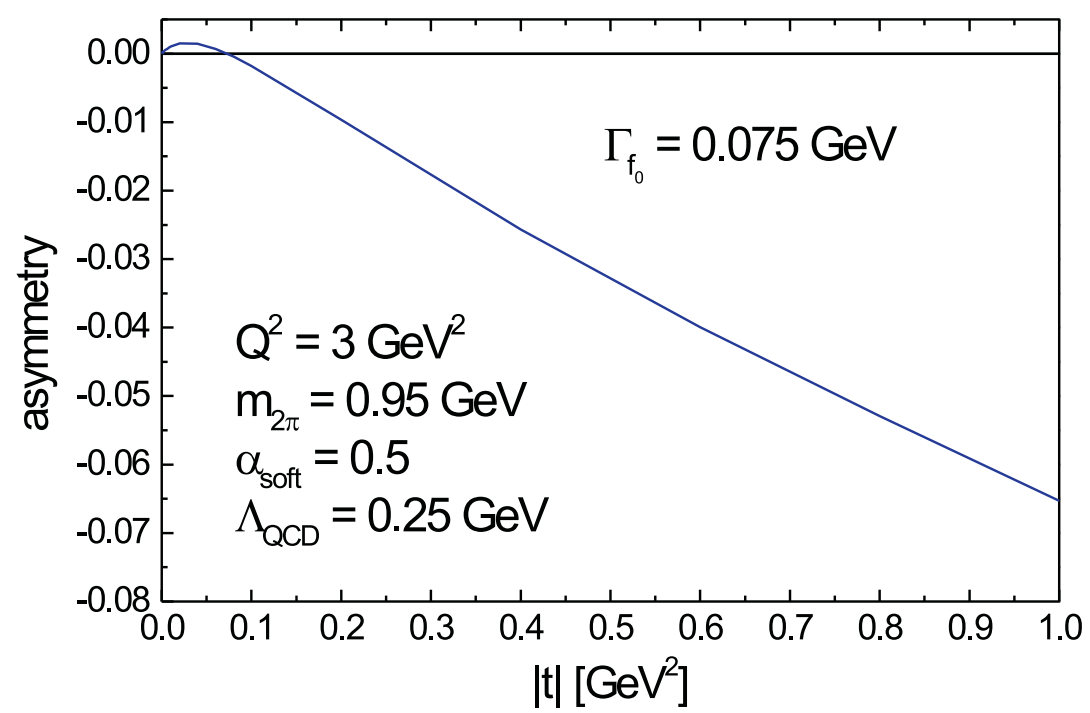

Figure 6: $t$-dependence of the asymmetry for $Q^{2}=3 \mathrm{GeV}^{2}$ and $m_{2 \pi}=0.95 \mathrm{GeV}$; the $f_{0}$ width has been taken to be $75 \mathrm{MeV}$.

by the experimental set-ups H1 [11] and ZEUS [29] at HERA. We are eagerly waiting for this confrontation of theory with data, which should lead us towards the discovery of the Odderon.

\section{Acknowledgements}

We acknowledge useful discussions with S. Brodsky, A.V. Efremov, I.F. Ginzburg, B. Loiseau, S.V. Mikhailov, B. Nicolescu, J. Olsson, T.N. Pham, A. Schäfer, T.N. Truong and S. Wallon.

This work is supported in part by the TMR and IHRP Programmes of the European Union, Contracts No. FMRX-CT98-0194 and No. HPRN-CT-2000-00130, RFFI Grant 16696, and iNTAS Project 587 (Call 2000).

\section{References}

[1] L. Lukaszuk and B. Nicolescu, Lett. Nuovo Cim. 8 (1973) 405

[2] J. Bartels, Nucl. Phys. B 151 (1979) 293; ibid B 175 (1980) 365;

J. Kwiecinski and M. Praszalowicz, Phys. Lett. B 94 (1980) 413.

[3] L.N. Lipatov, Phys. Lett. B 309 (1993) 394 ; JETP Lett. 59 (1994) 596 ; Sov. Phys. JETP Lett. 59 (1994) 571;

L.D. Fadeev and G.P. Korchemsky, Phys. Lett. B 342 (1995) 311

[4] R.A. Janik and J. Wosiek, Phys. Rev. Lett. 82 (1999) 1092 
[5] J. Bartels, L.N. Lipatov and G.P. Vacca, Phys. Lett. B 477 (2000) 178

[6] G. P. Korchemsky, J. Kotanski and A. N. Manashov, hep-ph/0111185.

[7] E.A. Kuraev, L.N. Lipatov and V.S. Fadin, Sov. JETP 44 (1976) 443 ; ibid 45 (1977) 199 ; Ya.Ya. Balitsky and L.N. Lipatov, Sov. J. Nucl. Phys. 28 (1978) 822 ; L.N. Lipatov, Sov. Phys. JETP 63 (1986) 904

[8] J. Czyzewski, J. Kwiecinski, L. Motyka and M. Sadzikowski, Phys. Lett. B 398 (1997) 400 ; erratum ibid B 411 (1997) 402

[9] R. Engel, D.Yu. Ivanov, R. Kirschner and L. Szymanowski, Eur. Phys. J. C 4 (1998) 93

[10] J. Bartels, M.A. Braun, D. Colferai and G.P. Vacca, Eur. Phys. J. C 20 (2001) 323

[11] J. Olsson (for the H1 Collab.), hep-ex/0112012

[12] E.R. Berger, A. Donnachie, H.G. Dosch, W. Kilian, O. Nachtmann and M. Reuter, Eur. Phys. J. C 9 (1999) 491

[13] H.G. Dosch, C. Ewerz and V. Schatz, hep-ph/0201294

[14] S.J. Brodsky, J. Rathsman and C. Merino, Phys. Lett. B 461 (1999) 114

[15] I.P. Ivanov, N.N. Nikolaev and I.F. Ginzburg, hep-ph/0110181

[16] M. Derrick et al, Phys. Lett. B356 (1995) 601; P. Amaudruz et al, Z. Phys. C51 (1991) 387; M. Arneodo et al, Nucl. Phys. B429 (1994) 503

[17] M. Diehl, T. Gousset, B. Pire and O.V. Teryaev, Phys. Rev. Lett. 81 (1998) 1782

[18] I.F. Ginzburg and D.Yu. Ivanov, Nucl. Phys. B 388 (1992) 376

[19] M. V. Polyakov and C. Weiss, Phys. Rev. D 59 (1999) 091502 arXiv:hep$\mathrm{ph} / 9806390$

M. V. Polyakov, Nucl. Phys. B 555 (1999) 231

[20] M. Diehl, T. Gousset and B. Pire, Phys. Rev. D 62 (2000) 073014

[21] B. Hyams et al., Nucl. Phys. B 64 (1973) 134

D.V. Bugg, B.S. Zou and A.V. Sarantsev, Nucl. Phys. B471 (1996) 59

R. Kaminski, L. Lesniak and K. Rybicki, Acta Phys.Polon. B 31 (2000) 895

[22] J.H. Kühn and A. Santamaria, Z. Phys. C 48 (1990) 445

[23] J.F. Gunion and D.E. Soper, Phys. Rev. D 15 (1977) 2617

[24] M. Fukugita and J. Kwiecinski, Phys. Lett. B 83 (1979) 119

[25] L. Frankfurt, W. Koepf and M. Strikman, Phys. Rev. D 54 (1996) 3194 
[26] B. Lehmann-Dronke, P. V. Pobylitsa, M. V. Polyakov, A. Schäfer and K. Goeke, Phys. Lett. B 475 (2000) 147 arXiv:hep-ph/9910310;

B. Lehmann-Dronke, A. Schäfer, M. V. Polyakov and K. Goeke, Phys. Rev. D 63 (2001) 114001 arXiv:hep-ph/0012108.

[27] O.V. Teryaev, T-odd fracture functions, in "SPIN-01", Proceedings of International Workshop, Dubna, 2002.

[28] B. Pire and O. V. Teryaev, Phys. Lett. B 496 (2000) 76

[29] J. Breitweg et al. [ZEUS Collaboration], Eur. Phys. J. C 6 (1999) 603 arXiv:hepex/9808020]. 